\title{
When Is a Functional Tree Transduction Deterministic?
}

\author{
Helmut Seidl \\ Fachbereich Informatik \\ Universität des Saarlandes \\ D-6600 Saarbrücken \\ Germany
}

\begin{abstract}
We give a decision procedure to determine whether or not the transduction of a functional transducer can be realized by a deterministic (resp. reduced deterministic) transducer. In case this is possible we exhibit a general construction to build this transducer.
\end{abstract}

\section{Introduction}

A (finite-state bottom-up) tree transducer is a finite state device. While traversing an input tree it produces an output tree in a bottom-up fashion. It is called functional iff there is at most one output tree for every input.

In practice, the concept of tree transducers is used, e.g., by tools for generating code selectors from formal descriptions of target machine languages (cf. [4]). Given a (regular) tree grammar which specifies the semantics of machine instructions in terms of operators of the intermediate representation language, a (typically non-deterministic) tree transducer is generated. The accepting computations describe all sequences of target machine code possibly generated for a given piece of program. Taking into account that different instructions may have different costs (e.g., need more or less processor cycles) a functional transducer is constructed which produces a cheapest instruction sequence.

Our main concern in this paper is the implementation of such functional transducers. Usually, this is done in two passes. The first one traverses the input tree to compute an overview over all possible computations from which the second one constructs an accepting computation and produces the output for it. We would like to avoid this second pass and produce output immediately. This can be done provided the corresponding tree transducer is deterministic. However, simple examples show that it is not always possible to construct an equivalent deterministic transducer. Therefore, the natural question arises: When can the transduction of a functional transducer be realized by a deterministic one?

Also, one would like to produce output only for subcomputations which finally is part of the resulting output. This can be guaranteed provided the tree transducer is reduced deterministic. Again, it is not always possible to construct even for a deterministic transducer an equivalent reduced deterministic transducer. Therefore, we also have to deal with the question: When can the 
iransduction of a functional transducer be realized by a reduced deterministic one?

Corresponding results for word transducers are known at least since [2]. Explicit constructions are described in [7] and [10].

So far, for tree transducers no related results are known. Several papers deal with deterministic or functional transducers, but they are mainly concerned with the equivalence problem. In [11] Zachar showed that for deterministic transducers equivalence is decidable. In [3] Engelfriet showed that it is decidable whether or not a tree transducer is functional. In [9] it was shown by the author that this problem is decidable even in polynomial time. As a corollary it follows that also equivalence of functional tree transducers is decidable in polynomial time provided the transducers agree in their domains. Especially, equivalence of unambiguous or deterministic transducers is decidable in polynomial time.

The present paper answers the question whether or not the transduction of a functional transducer can be realized by a deterministic or even reduced deterministic transducer. It is organized as follows. In Section 1, we provide some basic definitions. In Section 2, we introduce tree transducers. Especially, we allow these transducers to produce a final pattem after having reached a final state at the root of the input tree. This extension does not enlarge the power of a non-deterministic transducer; in case of a deterministic transducer this notion corresponds to the well-known class or "subsequential transducers" $[2,7,10]$ for words. Section 3 shows that, for every functional transducer we can construct an unambiguous transducer realizing the same transduction. Then we investigate deterministic transducers. Sections 4 and 5 present necessary conditions (D0), (D1) and (D2) (resp. (wD0), (D1) and (D2)) on a transducer for its transduction to be reduced deterministic (resp. deterministic). Our Main Theorems are presented in Section 6. Provided Properties (D0), (D1) and (D2) (resp. (wD0), (D1) and (D2)) hold for a functional reduced tree transducer $M$ we construct an equivalent reduced deterministic (resp. deterministic) transducer.

Because of space limitations most details of the proofs are omitted. They can be found in the full paper.

\section{Trees}

In this section we give basic definitions and state some fundamental properties about trees. Moreover, we present some technical propositions which will be used in the sequel.

A ranked alphabet or signature is a pair $(\Sigma, \rho)$ where $\Sigma$ is a finite alphabet and $\rho: \Sigma \rightarrow \mathbb{N}_{0}$ is a function mapping symbols to their rank. Usually, if $\rho$ is understood we write $\Sigma$ for short and define $\Sigma_{j}=\rho^{-1}(j) . T_{\Sigma}$ denotes the free $\Sigma$ algebra of (finite ordered $\Sigma$-labeled) trees, i.e., $T_{\Sigma}$ is the smallest set $T$ satisfying (i) $\Sigma_{0} \subseteq T$, and (ii) if $a \in \Sigma_{m}$ and $t_{1}, \ldots, t_{m} \in T$, then $a\left(t_{1}, \ldots, t_{m}\right) \in T$. Note: (i) can be viewed as the subcase of (ii) where $m=0$.

Let $N \subseteq \Sigma_{0}$. The $N$-depth of a tree $t \in T_{\Sigma}$, depth ${ }_{N}(t)$, is defined 
by $\operatorname{depth}_{N}(t)=\left\{\begin{array}{ll}-\infty & \text { if } t \in \Sigma_{0} \backslash N \\ 0 & \text { if } t \in N\end{array}\right.$ if $t \in \Sigma_{0}$, and $\operatorname{depth}_{N}(t)=1+$ $\max \left\{\operatorname{depth}_{N}\left(t_{1}\right), \ldots, \operatorname{depth}_{N}\left(t_{m}\right)\right\}$ if $t=a\left(t_{1}, \ldots, t_{m}\right)$ for some $a \in \Sigma_{m}, m>0$. If $N=\Sigma_{0}$ we omit the index $N$; also, if $N=\{x\}$ contains just one element we also write $\operatorname{depth}_{x}(-)$ instead of $\operatorname{depth}_{\{x\}}(-)$.

The size of $t,|t|$, is defined by $|t|=1$ if $t \in \Sigma_{0}$, and $|t|=1+\sum_{j=1}^{m}\left|t_{j}\right|$ if $t=a\left(t_{1}, \ldots, t_{m}\right)$ for some $a \in \Sigma_{m}, m>0$.

Let $X$ denote a set of variables of rank 0 . Define $T_{\Sigma}(X)=T_{\Sigma \cup X}$. We use this different notation in order to indicate which variables are to be substituted. (Clearly, $T_{\Sigma} \subseteq T_{\Sigma}(X)$.) $t \in T_{\Sigma}(X)$ is called $X$-proper iff every $x \in X$ occurs in $t$ exactly once. If $X=\{x\}$ we write $x$-proper instead of $\{x\}$-proper, and if $X$ is understood we skip the prefix $X$.

Every map $\theta: X \rightarrow T_{\Sigma}(X)$ can be extended to a map $\theta: T_{\Sigma}(X) \rightarrow$ $T_{\Sigma}(X)$ by $t \theta=x \theta$ if $t=x$, and $t \theta=a\left(t_{1} \theta, \ldots, t_{m} \theta\right)$ if $t=a\left(t_{1}, \ldots, t_{m}\right)$ with $a \in \Sigma . \theta$ is called $X$-substitution or simply substitution if $X$ is understood. If $X=\left\{x_{1}, \ldots, x_{m}\right\}$ and $x_{i} \theta=t_{i}$, we denote $t \theta$ also by $t\left[t_{1}, \ldots, t_{m}\right]$. Of special importance is the case where the set $X$ of variables which are to be substituted consists of just one element $x$. Assume $x \theta=t_{2}$ and $t_{1} \in T_{\Sigma}(x)=T_{\Sigma}(\{x\})$. Then we write $t_{1} \theta=t_{1} t_{2}$. The set $T_{\Sigma}(x)$ is a monoid w.r.t. $x$-substitution. (The neutral element is $x$ ). $T_{\Sigma}(x)$ is not a free monoid. Especially, $t_{1} t_{2}=t_{1}$ if $t_{1}$ does not contain an occurrence of $x$.

Let $\tilde{T}_{\Sigma}(x)$ denote the submonoid of $T_{\Sigma}(x)$ consisting of all trees $t$ which contain at least one occurrence of $x$. Note that trees in $\tilde{T}_{\Sigma}(x)$ may contain not only one occurrence of $x$ but also two occurrences or more. Call a tree $t \in \tilde{T}_{\Sigma}(x)$ $x$-irreducible iff $t \neq x$ and $t=u v$ implies either $u=x$ or $v=x$. If $x$ is understood we also skip the prefix $x$. So for example, $t=a(x, b(x))$ is irreducible whereas $t^{\prime}=a(b(x), b(x))=a(x, x) b(x)$ is not. Also, trees $a(x, t)$ or $a(t, x)$ for all trees $t \in T_{\Sigma}$ are irreducible. Let $I_{\Sigma}(x)$ denote the set of irreducible trees in $\tilde{T}_{\Sigma}(x)$. Note that $I_{\Sigma}(x)$ is infinite whenever $\Sigma_{j} \neq \emptyset$ for some $j>1$.

In [9] the following theorem was proven.

Theorem 1. As a monoid, $\tilde{T}_{\Sigma}(x)$ is frecly generated from $I_{\Sigma}(x)$, i.e., $\tilde{T}_{\Sigma}(x)=$ $I_{\Sigma}(x)^{*}$.

Consider for example the tree $t=a(a(b(x), b(x)), c)$ for $a, b, c \in \Sigma$. Then $t=$ $u_{1} u_{2} u_{3}$ for irreducible trees $u_{i}$ where $u_{1}=a(x, c) ; u_{2}=a(x, x)$ and $u_{3}=b(x)$.

Theorem 1 allows to define the $x$-length $|t|_{x}$ of a tree $t \in \tilde{T}_{\Sigma}(x):|t|_{x}=n$ iff $t=u_{1} \ldots u_{n}$ for irreducible trees $u_{j}$. Observe that $|t|_{x} \leq \operatorname{depth}_{x}(t)$, and $|t|_{x}=\operatorname{depth}_{x}(t)$ whenever $t$ is $x$-proper. If $t=u_{1} u_{2} \in \tilde{T}_{\Sigma}(x)$ then $u_{1}$ is called an $x$-prefix of $t$. Accordingly, $u_{2}$ is called $x-s u f f i x$ of $t$.

\section{Bottom-up Finite State Tree Transducers}

In this section we introduce finite tree automata (FTAs for short) and bottomup finite state tree transducers (FSTs for short). Similar to [9] we define an 
FST $M$ as a pair $(A, T)$ where $A$ is the finite tree automaton underlying $M$ and $T$ is the output function. Moreover, we recall the notion of reducedness of a transducer from $[8,9]$ which turns out to be useful in this context as well.

In the sequel, $X$ denotes the fix denumerable set $\left\{x_{i} \mid i \in \mathbb{N}\right\}$ of variables and for $m \geq 0, X_{m}=\left\{x_{1}, \ldots, x_{m}\right\}$.

A finite tree automaton (FTA for short) is a 4-tuple $A=\left(Q, \Sigma, \delta, Q_{F}\right)$ where $Q$ is a finite set of states, $Q_{F} \subseteq Q$ is the set of final states, $\Sigma$ is the signature of input trees, $\delta \subseteq \bigcup_{m>0} Q \times \Sigma_{m} \times Q^{m}$ is the set of transitions of $A$; the transitions in $\delta \cap \bigcup_{m \geq 0}\{q\} \times \Sigma_{m}^{\top} \times Q^{m}$ are also called q-transitions.

Let $t=a\left(t_{1}, \ldots, t_{m}\right) \in T_{\Sigma}\left(X_{k}\right)$ and $q, q_{1}, \ldots, q_{k} \in Q$. A $\left(q, q_{1} \ldots q_{k}\right)-$ computation $\phi$ of $A$ for $t$ starts at variables $x_{j}$ in states $q_{j}$ and consists of $\left(p_{j}, q_{1} \ldots q_{k}\right)$-computations of $A$ for the subtrees $t_{j}, j=1, \ldots, m$, together with a transition $\left(q, a, p_{1} \ldots p_{m}\right) \in \delta$ for the root. We write the state at the root to the left of the states at the variable leafs. This convention is chosen in accordance with our prefix notation of trees and the left-to-right order of substitutions. Formally, we represent $\phi$ as a tree over signature $\delta$ and set of variables $X_{k}$ as follows. If $t=x_{j}$ and $q=q_{j}$ then $\phi=x_{j}$. If $t=a\left(t_{1}, \ldots, t_{m}\right)$ then $\phi=\tau\left(\phi_{1}, \ldots, \phi_{m}\right)$ where $\tau=\left(q, a, p_{1} \ldots p_{m}\right) \in \delta$ for suitable states $p_{1}, \ldots, p_{m} \in Q$ and $\phi_{j}$ is a $\left(p_{j}, q_{1} \ldots q_{k}\right)$-computation for $t_{j}, j=1, \ldots, m$. Overloading the symbol $\delta$, we write $\left(q, t, q_{1} \ldots q_{k}\right) \in \delta$ iff there exists a $\left(q, q_{1} \ldots q_{k}\right)$-computation of $A$ for $t$.

Assume $t \in T_{\Sigma}\left(X_{\hat{k}}\right)$ and $t=t_{0}\left[t_{1}, \ldots, t_{k}\right]$. Assume $\phi_{0}$ is a $\left(q, p_{1} \ldots p_{k}\right)-$ computation for $t_{0}$, and $\phi_{i}$ are $\left(p_{i}, q_{1} \ldots q_{m}\right)$-computations for $t_{i}, i=1, \ldots, k$. Then $\phi_{0}\left[\phi_{1}, \ldots, \phi_{k}\right]$ is a $\left(q, q_{1} \ldots q_{m}\right)$-computation $\phi$ of $A$ for $t$. Conversely, if $t_{0}$ contains exactly one occurrence of any $x_{j}, j=1, \ldots, k$ (i.e., is $X_{k}$-proper), then every $\left(q, q_{1} \ldots q_{m}\right)$-computation $\phi$ for $t$ uniquely can be decomposed into a $\left(q, p_{1} \ldots p_{k}\right)$-computation $\phi_{0}$ for $t_{0}$, and $\left(p_{i}, q_{1} \ldots q_{m}\right)$-computations $\phi_{i}$ for $t_{i}$ (for suitable states $p_{i}$ ) such that $\phi=\phi_{0}\left[\phi_{1}, \ldots, \phi_{k}\right] . \phi_{i}$ is called subcomputation of $\phi$ on $t_{i}$.

A $(q, \epsilon)$-computation is also called $q$-computation. A $q$-computation is called accepting, iff $q \in Q_{F} \cdot \mathcal{L}(A)=\left\{t \in T_{\Sigma} \mid\right.$ there is an accepting computation of $A$ for $t\}$ is the language accepted by $A$.

$A$ is called unambiguous iff there is at most one accepting computation for every input tree $t \in T_{\Sigma} . A$ is called deterministic iff for every input symbol $a \in \Sigma$ and sequence $q_{1} \ldots q_{k} \in Q^{*}$, there is at most one state $q \in Q$ such that $\left(q, a, q_{1} \ldots q_{k}\right) \in \delta$. Clearly, every deterministic FTA is also unambiguous.

The size of $A,|A|$, is defined by

$$
|A|=\sum_{\left(q, a, q_{1} \ldots q_{m}\right) \in \delta}(m+2)
$$

A bottom-up finite state tree transducer (FST for short) is a pair $M=(A, T)$. $A=\left(Q, \Sigma, \delta, Q_{F}\right)$ is the FTA underlying $M$ whereas $T: \delta \cup Q_{F} \rightarrow T_{\Delta}(X)$, the output function of $M$, maps transitions and accepting states to their output patterns. Transitions $\tau=\left(q, a, q_{1} \ldots q_{m}\right)$ are mapped to trees $T(\tau) \in T_{\Delta}\left(X_{m}\right)$, whereas states $f \in Q_{F}$ are mapped to trees $T(f) \in T_{\Delta}\left(x_{1}\right)$. 
Note that an FST according to the definition in [9] is not allowed to produce output depending on the final state after having processed the whole input tree. For non-deterministic transducers, the additional feature does not increase the power of the corresponding device. For deterministic transducers it does. Here, it corresponds to the notion of "subsequential transducers" for words $[2,7,10]$.

$T$ is extended to computations as follows. Assume $\phi$ is a $\left(q, q_{1} \ldots q_{k}\right)$ computation of $A$. If $\phi=x_{j}$ for some $j$ then $T(\phi)=x_{j}$. If $\phi=\tau\left(\phi_{1}, \ldots, \phi_{m}\right)$ then $T(\phi)=T(\tau)\left[T^{\prime}\left(\phi_{1}\right), \ldots, T\left(\phi_{m}\right)\right] . T(\phi)$ is also called the output produced by $\phi$. By this definition, $T\left(\phi\left[\phi_{1}, \ldots, \phi_{k}\right]\right)=T(\phi)\left[T\left(\phi_{1}\right), \ldots, T\left(\phi_{k}\right)\right]$. Overloading the symbol $T$, we also write $\left(q, t, s, q_{1} \ldots q_{k}\right) \in T$ iff there exists a $\left(q, q_{1} \ldots q_{k}\right)^{-}$ computation $\phi$ of $A$ for $t$ with $T(\phi)=s$.

For some tree $t \in T_{\Sigma}, \mathcal{T}_{M}(t)=\{T(f) T(\phi) \mid \phi$ accepting $f$-computation of $A$ for $t\}$ denotes the set of outputs of $M$ for $t . \mathcal{T}(M)=\left\{(t, s) \mid t \in \mathcal{L}(A), s \in \mathcal{T}_{M}(t)\right\}$ is the transduction realized by $M . M$ is called functional iff $\# \mathcal{T}_{M}(t) \leq 1$ for every input tree $t \in T_{\Sigma} . M$ is called unambiguous or deterministic iff the underlying FTA is unambiguous or deterministic respectively. Clearly, every deterministic FST is unambiguous; and every unambiguous FST is functional.

The size $|M|$ of $M$ is defined by

$$
|M|=|A|+\sum_{\tau \in \delta}(|T(\tau)|+1)+\sum_{f \in Q_{F}}(|T(f)|+1)
$$

A state $q \in Q$ is called $u s e f u l$ iff an accepting computation $\phi$ exists that contains a $q$-transition. If this is not the case, $q$ is called useless. Useless states can be removed without changing the "behavior" of $A$ (and hence also $M$ ). An FTA $A$ is called reduced iff $A$ has no useless states.

The rest of this section is concerned with a "reduction" of transducers. Outputs for subcomputations which are not parts of the final output uniformly should equal a special output tree, namely $\perp$. $\perp$ is a new symbol (i.e., $\perp \notin \Delta$ ) of rank 0. Accordingly, we consider FSTs $M=(A, T)$ where the range of $T$ is contained in $T_{\Delta}(X) \cup\{\perp\}$. However, we consider in fact only FSTs $(A, T)$ where an output tree $\perp$ is always substituted for a variable $x_{j}$ which does not occur in the corresponding output pattern. Therefore, $\perp$ does not occur as the leaf of an output tree $s \neq \perp$, i.e., the output of every $\left(q, q_{1} \ldots q_{k}\right)$-computation $\phi$ of $A$ either equals $\perp$ or is in $T_{\Delta}\left(X_{k}\right)$.

The FST $M=(A, T)$ is called reduced, iff $A$ is reduced and there is some subset $U(M)$ of states such that (1) and (2) hold:

(1) For every $\tau=\left(q, a, q_{1} \ldots q_{m}\right) \in \delta$,

- $q \in U(M)$ iff $T(\tau)=\perp$; and

- $q_{j} \in U(M)$ iff $x_{j}$ does not occur in $T(\tau)$.

(2) $f \in Q_{F} \cap U(M)$ iff $T(f) \in T_{\Delta}$.

The states in $U(M)$ are exactly those which are used by subcomputations $\phi$ which produce $\perp$. If a subcomputation has reached some state not in $U(M)$ we can be sure that the output for the corresponding subcomputation is part of the final output. The proof of a corresponding proposition in [8] can be carried over to prove: 
Proposition 2. Assume $M$ is an FST. Then a reduced FST $M_{r}$ can be constructed in polynomial time such that

- $\mathcal{T}(M)=\mathcal{T}\left(M_{r}\right)$;

$-\left|M_{r}\right| \leq 2 \cdot|M|$.

Proof. We repeat the construction of $M_{r}$ since we will refer to it in Section 6 .

Define $\bar{M}=(\bar{A}, \bar{T})$ where $\bar{A}=\left(\bar{Q}, \Sigma, \bar{\delta}, \bar{Q}_{F}\right)$ where $\bar{Q}=Q \times\{0,1\} ; \bar{Q}_{F}$ consists of all pairs $\langle f, i\rangle$ where $f \in Q_{F}$ and $i=0$ iff $x_{1}$ does not occur in $T(f)$. For such a final state $\langle f, i\rangle$ define $\tilde{T}(\langle f, i\rangle)=T(f)$. Finally, $\bar{\delta}$ and $\bar{T} \mid \bar{\delta}$ are defined as follows. Assume $\tau=\left(q, a, q_{1} \ldots q_{k}\right) \in \delta$.

Then $\langle\tau, 0\rangle=\left(\langle q, 0\rangle, a,\left\langle q_{1}, 0\right\rangle \ldots\left\langle q_{m}, 0\right\rangle\right),\langle\tau, 1\rangle=\left(\langle q, 1\rangle, a,\left\langle q_{1}, \epsilon_{1}\right\rangle \ldots\left\langle q_{m}, \epsilon_{m}\right\rangle\right)$ are in $\bar{\delta}$ where $\epsilon_{j}=1$ if $x_{j}$ occurs in $T(\tau)$, and $\epsilon_{j}=0$ otherwise. $\langle\tau, 1\rangle$ and $\langle\tau, 0\rangle$ are the versions of $\tau$ which are used in subcomputations whose output will be part resp. will not be part of the final output. Therefore, we put $\bar{T}(\langle\tau, 1\rangle)=T(\tau)$ and $\bar{T}((\tau, 0))=1$.

Then, $\mathcal{T}(M)=\mathcal{T}(\bar{M})$ and $\vec{M}$ satisfies (1) and (2) of the definition of reducedness with $U(\bar{M})=Q \times\{0\}$. Now eliminate superfluous states and transitions. Note here that by definition, every state of $\vec{M}$ is derivable; however, not every state is also accessible from $Q_{r, F}$.

Prop. 2 is the justification that we always w.1.o.g. may assume that a functional tree transducer is reduced. However, if the tree transducer $M$ was deterministic it is not necessarily the case that the reduced tree transducer $M_{r}$ constructed according to Prop. 2 is deterministic as well!

\section{Unambiguous Transducers}

As for word transducers, for every functional transducer we can construct a reduced unambiguous transducer realizing the same transduction. We have:

Theorem 3. For every functional transducer $M$ an unambiguous transducer $M_{u}$ can be constructed such that

1. $\mathcal{T}(M)=\mathcal{T}\left(M_{u}\right)$;

2. $\left|M_{u}\right| \leq|M| \cdot 3^{|M|}$.

Proof. The idea is the following. First, we construct an FTA $A_{0}$ whose states are pairs $\langle D, B\rangle$ of sets of states of $A$ where $D$ is the set of derivable states and $B \subseteq D$ is the set of accessible states in $D$. Then we assume a natural ordering on the states of $A$. This ordering induces a lexicographical ordering on every subset of $Q^{k}, k \geq 0$. We want to produce output according to an accepting computation of $A$ for the given input tree $t$ which is determined by the following strategy: for every node of $t$ we select a unique accessible and derivable state together with a transition in a topdown fashion as follows. We start at the root by selecting the smallest accepting state which is derivable. Now assume transition $\tau=\left(\langle D, B\rangle, a,\left\langle D_{1}, B_{1}\right\rangle \ldots\left\langle D_{k}, B_{k}\right\rangle\right)$ has been chosen at some node 
$o$ of the accepting computation $\phi$ of $A_{0}$ for the input tree $t$, and the selected state for node $o$ is $q$. By construction, $q \in B$. Let $\operatorname{trans}(\tau, q)$ denote the set of $q$-transitions $\left(q, a, q_{1}^{\prime} \ldots q_{k}^{\prime}\right)$ of $A$ such that $q_{j}^{\prime} \in D_{j}$ for all $j$. By construction of $A_{0}, \operatorname{trans}(\tau ; q)$ is not empty. Then the transition of $\phi$ for $o$ is that element of $\operatorname{trans}(\tau, q)$ for which the sequence $q_{1} \ldots q_{k}$ of successor states is minimal, and select for $j=1, \ldots, k$, state $q_{j}$ for the $j$-th successor node of $o$ in $t$. By construction, $\phi$ is an accepting computation of $A$ for $t$. Finally, the output we produce for $t$ is the output of $A$ for $\phi$.

To implement this idea we construct an FST $M_{1}=\left(A_{1}, T_{1}\right)$ where $A_{1}$ is obtained by adding a third component to the states of $A_{0}$ holding the corresponding selected states. Formally, $A_{1}=\left(Q_{1}, \Sigma, \delta_{1}, Q_{1, F}\right)$ where $Q_{1}=\{\langle D, B, q\rangle \mid q \in$ $B \subseteq D\}$ with $Q_{1, F}=\left\{\langle D, B, q\rangle \in Q_{1} \mid B=Q_{F} \cap D, q \in B\right.$ minimal $\}$ where $T_{1}(\langle D, B, q\rangle)=T(q)$. The transition relation $\delta_{1}$ is defined as follows.

$\tau=\left(\langle D, B, q\rangle, a\left\langle D_{1}, B_{1}, q_{1}\right\rangle \ldots\left\langle D_{k}, B_{k}, q_{k}\right\rangle\right) \in \delta_{1}$ iff

- $D=\left\{p \in D \mid \forall j \equiv p_{j} \in D_{j}:\left(p, a, p_{1} \ldots p_{k}\right) \in \delta\right\} ;$

- $B_{j}=\left\{p_{j} \in D_{j} \mid \exists p \in B, \forall j^{\prime} \neq j \exists p_{j^{\prime}} \in D_{j^{\prime}}:\left(p, a, p_{1} \ldots p_{k}\right) \in \delta\right\}$;

- and $q_{1} \ldots q_{k} \in B_{1} \times \ldots \times B_{k}$ is minimal such that $\tau_{0}=\left(q, a, q_{1} \ldots q_{k}\right) \in \delta$.

Moreover, $T_{1}(\tau)=T\left(\tau_{0}\right)$.

Finally, $M_{u}$ is obtained from $M_{1}$ by removing useless states and transitions from $A_{1}$ and restricting $T_{1}$ accordingly.

\section{Cyclic Computations}

In this section we recall the notion of a pairing. The notion of a pairing was introduced in [9] to allow for an elegant description of pairs of computations of a transducer for the same input tree. We use it to state the necessary conditions (D0) (resp. (wD0)) and (D1) for an FST such that $\mathcal{T}(M)$ can be realized by a reduced deterministic (resp. deterministic) transducer.

Given an FTA $A=\left(Q, \Sigma, \delta, Q_{F}\right)$ we define the FTA $A^{(2)}$ as follows. Let $\bar{A}=\left(\bar{Q}, \Sigma, \bar{\delta}, \bar{Q}_{F}\right)$ be the FTA with $\bar{Q}=Q^{2}$ and $\bar{Q}_{F}=Q^{2}$ where $\tau=\left(\langle p, q\rangle, a,\left\langle p_{1}, q_{1}\right\rangle \ldots\left\langle p_{k}, q_{k}\right\rangle\right) \in \bar{\delta}$ iff $\tau_{1}=\left(p, a, p_{1} \ldots p_{k}\right) \in \delta$ and $\tau_{2}=$ $\left(q, a, q_{1} \ldots q_{k}\right) \in \delta$. In this case, we write $\tau=\left\langle\tau_{1}, \tau_{2}\right\rangle$. Accordingly, computations of $\vec{A}$ are viewed as pairs of computations of $A$ for the same input tree.

$\bar{A}$ need not be reduced. Thus, $A^{(2)}$ is obtained from $\bar{A}$ by removing useless states and transitions. In $A^{(2)}$ every pair of states is accepting which jointly are derivable.

A triple $\Pi=\left(A, T_{1}, T_{2}\right)$ is called pairing iff $A$ is an FTA and both $T_{1}$ and $T_{2}$ are output functions for $A$. In our applications here $\left(A, T_{i}\right)$ always will be functional.

For $M$ we define the pairing $M^{(2)}=\left(A^{(2)}, T_{1}, T_{2}\right)$ where $T_{i}$ produce the output according to the $i$-th components of the transitions of $\delta^{2}$ with $T_{i}\left(z^{\prime}\right)=x_{1}$ for all $z^{\prime}$. 
A proper $\left(z^{\prime}, z^{\prime}\right)$-computation $\phi$ is called cyclic iff both $T_{1}(\phi) \notin\left\{1, x_{1}\right\}$ and $T_{2}(\phi) \notin\left\{\perp, x_{1}\right\}$. A state $z^{\prime}$ of $A^{(2)}$ is called cyclic iff a cyclic $\left(z^{\prime}, z^{\prime}\right)$ computation exists. Cyclic computations play an essential role in our characterization of (reduced) deterministic transductions. Property (D0) says that a $(z, z)$-computation already is cyclic provided either the first or the second output for it is non-trivial:

$M$ has Property (D0) iff

(D0) $T_{1}(\psi) \in\left\{\perp, x_{1}\right\}$ iff $T_{2}(\psi) \in\left\{\perp, x_{1}\right\}$ for every proper $(z, z)$-computation $\psi$ of $A^{(2)}$.

It turns out that although Property (D0) is always satisfied when $\mathcal{T}(M)$ can be realized by a reduced deterministic transducer (cf. Prop. 4 (1)), it may not be satisfied when $\mathcal{T}(M)$ can be realized by a deterministic transducer only. Therefore, a weaker property (wD0) is needed. (wD0) limits the possibilities how $M$ may violate (D0):

$M$ has Property (wDo) iff

(wD0) The set of states of $A^{(2)}$ is the (disjoint) union of sets $Q_{0}, Q_{1}, Q_{2}$ and $Q_{3}$ where

- For every $z^{\prime} \in Q_{0}$ and every proper $\left(z^{\prime}, z^{\prime}\right)$-computation $\psi$ of $A^{(2)}$, $T_{i}(\psi) \in\left\{L, x_{1}\right\}$ for $i=1,2$.

- For every $z^{\prime} \in Q_{1}$ the first output of every proper $\left(z^{\prime}, z^{\prime}\right)$-computation of $A^{(2)}$ is in $\left\{\perp, x_{1}\right\}$, and some $\left(z^{\prime}, z^{\prime}\right)$-computation $\psi$ of $A^{(2)}$ exists such that $T_{2}(\psi) \notin\left\{\perp, x_{1}\right\}$.

- For every $z^{\prime} \in Q_{2}$ the second output of every proper $\left(z^{\prime}, z^{\prime}\right)$-computation of $A^{(2)}$ is in $\left\{\perp, x_{1}\right\}$, and some $\left(z^{\prime}, z^{\prime}\right)$-computation $\psi$ of $A^{(2)}$ exists such that $T_{1}(\psi) \notin\left\{\perp, x_{1}\right\}$.

- For every $z^{\prime} \in Q_{3}$ and every proper $\left(z^{\prime}, z^{\prime}\right)$-computation $\psi$ of $A^{(2)}$, $T_{1}(\psi) \in\left\{\perp, x_{1}\right\}$ iff $T_{2}(\psi) \in\left\{\perp, x_{1}\right\}$, and some proper $\left(z^{\prime}, z^{\prime}\right)$ computation $\psi^{\prime}$ of $A^{(2)}$ exists such that $T_{i}\left(\psi^{\prime}\right) \notin\left\{1, x_{1}\right\}$.

For these sets the following holds:

1. If for some $i \in\{1,2,3\}$, some state $z_{2}$ of $A^{(2)}$ is reachable from some $z_{1} \in Q_{i}$ then $z_{2} \in Q_{0} \cup Q_{i}$.

2. If for some $i \in\{1,2,3\}$, some state $z_{2} \in Q_{i}$ is reachable from some $z_{1}$ of $A^{(2)}$ then $z_{1} \in Q_{0} \cup Q_{i}$.

3. Assume $\left(z, a, z_{1} \ldots z_{k}\right)$ is a transition $A^{(2)}$ and for some $i \in\{1,2,3\}$ and $j$, some state of $Q_{i}$ is reachable from $z_{j}$. Then every state reachable from some $z_{j^{\prime}}, j^{\prime}=1, \ldots, k$, is in $Q_{0} \cup Q_{i}$.

Proposition 4. Assume $M=(A, T)$ is a reduced FST.

1. If $\mathcal{T}(M)$ can be realized by a reduced deterministic transducer then $M$ has Property $(D O)$.

D. If $\mathcal{T}(M)$ can be realized by a deterministic transducer then $M$ has Property $(w D 0)$. 
It can be decided in polynomial time whether or not $M$ has Property (D0) or (wDo).

Property (D1) speaks on the difference between the produced outputs when reaching a cyclic state $z$.

$M$ has Property (D1) iff

(D1) For every state $z$ of $A^{(2)}$, every $z$-computation $\phi$ and every cyclic $(z, z)-$ computation $\psi$,

(1) $r_{1}, r_{2} \in \tilde{T}_{\Delta}\left(x_{1}\right)$ exist with $x_{1} \in\left\{r_{1}, r_{2}\right\}$ with $r_{1} T_{1}\left(\psi^{k} \phi\right)=r_{2} T_{2}\left(\psi^{k} \phi\right)$ for every $k \geq 0$.

For words, Property (D0) together with Property (D1) hold iff all pairs of states are twinned (cf. [2, 10]). Property (wD0) is without analogue in the word case. Assume $M$ has Property (D1). Then also the following Properties hold:

(2) $r_{i}$ is a prefix of $T_{3-i}\left(\psi^{l}\right)$ for some $l \geq 0$ with $\operatorname{depth}\left(r_{i}\right) \leq \operatorname{depth}\left(T_{3-i}(\phi)\right)$.

(3) $\left|T_{1}(\psi)\right|_{x_{1}}=\left|T_{2}(\psi)\right|_{x_{1}}$.

(4) Assume $\psi^{\prime}$ is another $(z, z)$-computation of $A^{(2)}$. Then also $r_{1} T_{1}\left(\left(\psi^{\prime}\right)^{k} \phi^{\prime}\right)=r_{2} T_{2}\left(\left(\psi^{\prime}\right)^{k} \phi\right)$ for every $k \geq 0$.

(5) Assume $\phi^{\prime}$ is another $z$-computation of $A^{(2)}$, and $r_{1}^{\prime} T_{1}\left(\psi^{k} \phi^{\prime}\right)=$ $r_{2}^{\prime} T_{2}\left(\psi^{k} \phi^{\prime}\right)$ for every $k \geq 0$. Then for $i=1,2, r_{i}^{\prime} s_{i}^{\prime}=r_{i} s_{i}$ for $s_{i}, s_{i}^{\prime} \in$ $\tilde{T}_{\Delta}\left(x_{1}\right)$ with $x_{1} \in\left\{s_{i}^{\prime}, s_{i}\right\}$.

Especially by (5), $r_{1}$ and $r_{2}$ are uniquely determined.

Proposition 5. Assume for $j=1,2, \psi_{j}$ is a cyclic $\left(z_{j}, z_{j}\right)$-computation of $A^{(2)}$, $\phi_{1}$ is a $\left(z_{1}, z_{2}\right)$-computation and $\phi_{2}$ is a $z_{2}$-computation.

If $M$ has Property (D1) then for every $k \geq 0$,

$$
r_{1} T_{1}\left(\psi_{2}^{k} \phi_{2}\right)=r_{1} T_{1}\left(\psi_{2}^{k} \phi_{2}\right)
$$

and also

$$
s_{1} T_{1}\left(\psi_{1}^{k} \phi_{1}\right) r_{2}=s_{2} T_{2}\left(\psi_{1}^{k} \phi_{1}\right) r_{1}
$$

for some $s_{i}, r_{i} \in \tilde{T}_{\Delta}\left(x_{1}\right)$ where both $x_{1} \in\left\{s_{1}, s_{2}\right\}$ and $x_{1} \in\left\{r_{1}, r_{2}\right\}$.

Proposition 6. Let $M$ be a reduced FST. If $T(M)$ can be realized by a deterministic transducer then $M$ has Property (D1).

It turns out that Property (D1) together with Property (D0) (or (wD0)) neither characterizes determinism nor reduced determinism. 


\section{Bounded Difference}

In this section we introduce Property (D2) which completes the set of properties necessary to characterize deterministic resp. reduced deterministic transductions. Property (D2) has no counterpart in the word case. Together Properties (D0), (D1) and (D2) (resp. (wD0), (D1) and (D2)) allow to prove that $M$ is of bounded difference (bd) (resp. weakly bounded difference (wbd)). In the next section we will construct an equivalent reduced deterministic transducer for $M$ whenever $M$ is of bounded difference thus showing that Property (bd) (and hence jointly Properties (D0), (D1) and (D2)) is also sufficient for reduced determinism. A corresponding result holds for determinism as well where Properties (D0) and (bd) are replaced by Properties (wD0) and (wbd) respectively.

$M$ has Property (D2) iff for every transition $\tau=\left(z, a, z_{1} \ldots z_{k}\right)$ and $z_{j}-$ computations $\phi_{j}$ of $A^{(2)}, j=1, \ldots, k$, the following holds.

(D2) Let $J$ denote the set of all $x_{j}$ where $\phi_{j}=\psi_{j 1} \psi_{j 2} \psi_{j 3}$ for proper computations $\psi_{j i}$ where $\psi_{j 2}$ is cyclic. Let $y_{i}=T_{i}(\tau)\left[v_{i 1}, \ldots, v_{i k}\right]$ where $v_{i j}=x_{j}$ whenever $x_{j} \in J$ and $v_{i j}=T_{i}\left(\phi_{j}\right)$ otherwise. Assume $\# J>1$. Consider the decompositions $y_{i}=r_{i} y_{i}^{\prime}$ where $r_{i}$ is the maximal $x_{1}$-prefix of $y_{i}$. Then $J$-substitutions $\theta_{i}$ exit such that for every $j \in J$,

1. $x_{j} \in\left\{x_{j} \theta_{1}, x_{j} \theta_{2}\right\}$;

2. $y_{1}^{\prime} \theta_{2}=y_{2}^{\prime} \theta_{1}$

3. $\left(x_{j} \theta_{1}\right) T_{1}\left(\psi_{j 1} \psi_{j 2}^{m} \psi_{j 3}\right)=\left(x_{j} \theta_{2}\right) T_{2}\left(\psi_{j 1} \psi_{j 2}^{m} \psi_{j 3}\right)$ for every $m \geq 0$.

Analogously to Prop. 5 we have:

Proposition 7. Let $M$ be a reduced FST satisfying properties (D1) and (D2). Assume the assumptions of Property (DQ) hold, and additionally a cyclic $\left(z^{\prime}, z^{\prime}\right)$ computation $\psi^{\prime}$ exists for some state $z^{\prime}$ together with a proper $\left(z^{\prime}, z\right)$-computation $\phi^{\prime}$. Then some $s_{i} \in \tilde{T}_{\Delta}\left(x_{1}\right)$ exist with $x_{1} \in\left\{s_{1}, s_{2}\right\}$ such that $s_{1} T_{1}\left(\left(\psi^{\prime}\right)^{m} \phi^{\prime}\right) r_{1}=$ $s_{2} T_{2}\left(\left(\psi^{\prime}\right)^{m} \phi^{\prime}\right) r_{2}$ for every $m \geq 0$.

Note especially, that in the situation of Prop. 7 , either $r_{1}$ is a suffix of $r_{2}$ or vice versa.

Proposition 8. Let $M$ be a reduced FST. If $\mathcal{T}(M)$ can be realized by a deterministic transducer then $M$ has Property (DQ2).

For the following always assume that $A$ has $n>0$ states. The reduced FST $M$ is of bounded difference (bd) iff the following holds:

(1) For every $z$-computation $\phi$ of $A^{(2)}$ one of the following statements hold:

1. depth $T_{i}(\phi)<n \cdot|M|$ for both $i=1$ and $i=2$;

2. $T_{i}(\phi)=v_{i} u$ for some $u \in T_{\Delta}$ and $v_{i} \in \tilde{T}_{\Delta}\left(x_{1}\right)$ such that for $i=1,2$, depth $_{x_{1}}\left(v_{i}\right)<n \cdot|M|$ and $v_{i}$ does not contain subtrees in $T_{\Delta}$ of depth at least $(n+1) \cdot|M|$. 
(2) For every transition $\tau=\left(z, a, z_{1} \ldots z_{k}\right)$ and $z_{j}$-computations $\phi_{j}$ of $A^{(2)}$ the following holds. Let $J_{i}$ denote the set of all $x_{j}$ where $\operatorname{depth}\left(T_{i}\left(\phi_{j}\right)\right) \geq n \cdot|M|$ for $i=1,2$, and $J=J_{1} \cup J_{2}$. Let $y_{i}=T_{i}(\tau)\left[v_{1 i}, \ldots, v_{k i}\right]$ where $v_{j i}=x_{j}$ whenever $x_{j} \in J$ and $v_{j i}=T_{i}\left(\phi_{j}\right)$ otherwise. Assume \#J>1. Consider the decompositions $y_{i}=z_{i} y_{i}^{\prime}$ where $z_{i}$ is the maximal $x_{1}$-prefix of $y_{i}$. Then $J$-substitutions $\theta_{i}, i=1,2$, exist such that for every $j \in J$,

1. $x_{j} \in\left\{x_{j} \theta_{1}, x_{j} \theta_{2}\right\} \subseteq \tilde{T}_{\Delta}\left(x_{j}\right)$;

2. $y_{1}^{\prime} \theta_{2}=y_{2}^{\prime} \theta_{1}$

3. $\left(x_{j} \theta_{1}\right) T_{1}\left(\phi_{j}\right)=\left(x_{j} \theta_{2}\right) T_{2}\left(\psi_{j}\right)$.

$M$ is of weakly bounded difference ( $w b d$ ) iff for every $z$-computation $\phi$ of $A^{(2)}$, statement (1) must only hold provided depth $T_{i}(\phi) \geq n \cdot|M|$ for both $i \in\{1,2\}$; and statement (2) only provided $J_{1} \neq \emptyset \neq J_{2}$.

\section{Characterizations}

This section presents the main theorems of this paper. Theorem 9 states that the transduction of a reduced functional transducer $M$ can be realized by a reduced deterministic one iff $M$ has Properties (D0), (D1) and (D2). Theorem 10 is the corresponding version for realization by deterministic transducers. Here, the characterization only differs in that Property (D0) is replaced by Property (wD0).

Theorem 9. Assume $M$ is a reduced functional FST. Then the following three statements are equivalent:

(1) $\mathcal{T}(M)$ can be realized by a reduced deterministic transducer.

(2) $M$ has Properties (D0), (D1) and (D2).

(3) $M$ is of bounded difference.

It can be decided whether or not the transduction of an FST can be realized by a reduced deterministic one.

Proof. Let $M=(A, T)$ be a reduced functional FST where $A=\left(Q, \Sigma, \delta, Q_{F}\right)$ and $n=\# Q>0$. We only give the construction showing that (3) implies (1). Assume that $M$ is of bounded difference. We construct a reduced deterministic FST $M_{d}$ realizing $\mathcal{T}(M)$.

Let $A_{0}=\left(Q_{0}, \Sigma, \delta_{0}, Q_{0, F}\right)$ be the standard subset automaton for $A$, i.e., $Q_{0}=2^{Q} ; Q_{0, F}=\left\{B \subseteq Q \mid B \cap Q_{F} \neq \emptyset\right\} ;$ and $\delta_{0}$ consists of all transitions $\left(B, a, B_{1} \ldots B_{k}\right)$ where $B=\left\{q \in Q \mid \forall j \exists q_{j} \in B_{j}:\left(q, a, q_{1} \ldots q_{k}\right) \in \delta\right\}$.

The idea of the construction is the following. When starting the computation for some input tree $t$ at the leaves, $M_{d}$ behaves like $A_{0}$ but records for every accessible state $q$ that part of the output of some $q$-computation of which it is not yet certain whether it will be part of the final output or not. This is similar as in the word case. The new difficulty that arises is to handle the start-up phase. Having accumulated sufficiently large possible output trees we have to find the right "end", i.e., that subtree which safely can be produced and leave variables 
at the right places. This choice is easy if for more than one of the subtrees at the present node large outputs can be produced (cf. Case 3). Otherwise, the size of the subtree's output must be large enough such that it can be uniquely detected (cf. Case 1).

Formally, we construct $M_{d}=\left(A_{d}, T_{d}\right)$ with $A_{d}=\left(Q_{d}, \Sigma, \delta_{d}, Q_{d, F}\right)$ as follows.

$Q_{d}$ is some set of mappings $\mu: B \rightarrow T_{\Delta}\left(x_{1}\right) \cup\{\perp\}, B \in Q_{0}$ such that $\mu(q)$ is a tree of depth less than $(2 n+1) \cdot|M| Q_{d, F}$ consists of all mappings $\mu \in Q_{d}$ which are defined for some $q \in Q_{F}$ where $T_{d}(\mu)=T(q) \mu(q)$, and $U\left(M_{d}\right)=\left\{\mu \in Q_{d} \mid \operatorname{range}(\mu) \subseteq T_{\Delta} \cup\{\perp\}\right\}$.

The sets $Q_{d}$ and $\delta_{d}$ are iteratively determined as the union of their "approximations", i.e., by

$$
Q_{d}=\bigcup_{\nu \geq 0} Q^{(\nu)} \text { and } \delta_{d}=\bigcup_{\nu \geq 0} \delta^{(\nu)}
$$

where $Q^{(0)}=\emptyset$ and $\delta^{(0)}=\emptyset$. The construction will be done such that the following invariant holds:

(0) Assume $(\mu, u, s, \epsilon) \in T_{d}$, and $B$ is the domain of $\mu$. Then $(B, u, \epsilon) \in \delta_{0}$, and for every $q \in B$,

- $(q, u, \mu(q) s, \epsilon) \in T$

- $x_{1}$ occurs in $\mu(q)$ iff $x_{1}$ occurs in $\mu\left(q^{\prime}\right)$ for every $q^{\prime} \in B$;

- If $x_{1}$ occurs in $\mu(q)$ then $\operatorname{depth}(s) \geq(n+1) \cdot|M|$. Otherwise, $s=\perp$.

Assume $\nu>0$ and the sets $Q^{(\nu-1)}$ and $\delta^{(\nu-1)}$ already have been defined. Let $\mu_{1}, \ldots, \mu_{k} \in Q^{(\nu-1)}, B_{j}$ be the domains of $\mu_{j}, j=1, \ldots, k$, and $\left(B, a, B_{1} \ldots B_{k}\right)$ a transition of the subset automaton $A_{0}$. We construct one map $\mu: B \rightarrow T_{\Delta}\left(x_{1}\right) \cup$ $\{\perp\}$ such that $\mu \in Q^{(j)}$ and $\tau=\left(\mu, a, \mu_{1} \ldots \mu_{k}\right) \in \delta^{(j)}$ and define $T_{d}(\tau)$.

By definition of $\delta_{0}$, for every $q \in B$ some $\tau_{q}=\left(q, a, p_{q, 1} \ldots p_{q, k}\right) \in \delta$ exists with $p_{q, j} \in B_{j}$ for all $j$. For $q \in B$ let $u_{q}=T\left(\tau_{q}\right)\left[\mu_{1}\left(p_{q, 1}\right) x_{1}, \ldots, \mu_{k}\left(p_{q, k}\right) x_{k}\right]$, and let $J_{q}$ denote the set of all $j$ such that either $\mu_{j}\left(p_{q, j}\right) \in \tilde{T}_{\Delta}\left(x_{1}\right)$ or has depth $\geq n \cdot|M|$.

Case 1: $u_{r} \in T_{\Delta}$ for some $r \in B$.

According to claim $(0)$ for $Q^{(\nu-1)}, u_{q} \in T_{\Delta}$ for all $q \in B$. If $\operatorname{depth}\left(u_{q}\right)<$ $(2 n+1) \cdot|M|$ for all $q \in B$ then we define $\mu(q)=u_{q}$ and $T_{d}(\tau)=\perp$.

Otherwise, some $r \in B$ exists with $\operatorname{depth}\left(u_{r}\right) \geq(2 n+1) \cdot|M|$. We claim that

(1) $v \in T_{\Delta}$ and $w_{q} \in \tilde{T}_{\Delta}\left(x_{1}\right)$ exist such that for all $q \in B$,

- $u_{q}=w_{q} v$;

- $\operatorname{depth}_{x_{1}}\left(w_{q}\right)<n \cdot|M|$;

- $w_{q}$ does not contain subtrees from $T_{\Delta}$ of depth $\geq(n+1) \cdot|M|$;

- the maximal common $x_{1}$-suffix of $w_{q}, q \in B$, is $x_{1}$.

Under this assumption, we put $\mu(q)=w_{q}$ and $T_{d}(\tau)=v$.

Case 2: $\exists j: \mu_{j}\left(p_{r, j}\right) \in \tilde{T}_{\Delta}\left(x_{1}\right)$ for some $r \in Q$ and $\forall q \in B: \# J_{q} \leq 1$.

Then by claim $(0)$ for $Q^{(\nu-1)}, \mu_{j}\left(p_{q, j}\right) \in \tilde{T}_{\Delta}\left(x_{1}\right)$ for every $q \in B$. Hence, $J_{q}=\{j\}$, and $\mu_{j^{\prime}}\left(p_{q, j^{\prime}}\right) \in T_{\Delta}$ for all $j^{\prime} \neq j$. Therefore, $u_{q} \in \tilde{T}_{\Delta}\left(x_{j}\right)$ for every 
$q \in B$. Moreover, $u_{q}$ does not contain subtrees in $T_{\Delta}$ of depth $\geq(n+1) \cdot|M|$. Let $u_{q}=w_{q} y$ where $y$ is the maximal common $x_{j}$-suffix of all $u_{q}$. We claim that

(2) For every $q \in B$, $\operatorname{depth}_{x_{1}}\left(w_{q}\right)<n \cdot|M|$.

Provided Claim (2) holds, we put $\mu(q)=w_{q} x_{1}$ and $T_{d}(\tau)=y$.

Case 3: $\exists j: \mu_{j}\left(p_{r, j}\right) \in \tilde{T}_{\Delta}\left(x_{1}\right)$ and $\# J_{r}>1$ for some $r \in B$.

Again by Claim $(0)$ for $Q^{(\nu-1)}, \mu_{j}\left(p_{q, j}\right) \in \tilde{T}_{\Delta}\left(x_{1}\right)$ for all $q \in B$. We claim:

(3) There exists exactly one $s \in T_{\Delta}\left(X_{k}\right)$ such that the following holds:

- for all $q \in B, u_{q}=v_{q} s$ where $v_{q} \in \tilde{T}_{\Delta}\left(x_{1}\right)$ with $\operatorname{depth}_{x_{1}}\left(v_{q}\right)<n \cdot|M|$ such that $v_{q}$ does not contain subtrees from $T_{\Delta}$ of depth $\geq(n+1) \cdot|M|$;

- $s$ is maximal with this property.

Provided Claim (3) holds, we define $\mu(q)=v_{q}$ for every $q \in B$, and put $T_{d}(\tau)=s$. Observe that $x_{j}$ occurs in $s$ iff $\mu_{j}\left(p_{q, j}\right) \notin T_{\Delta}$ for some $q$. Therefore, (provided claim (0) for $Q^{(\nu-1)}$ holds) $x_{j}$ occurs in $T_{d}(\tau)$ iff $\mu_{j} \notin U\left(M_{d}\right)$.

This finishes the construction. In order to prove its correctness we claim:

(4) Assume $(B, u, \epsilon) \in \delta_{0}$. Then $(\mu, u, s, \epsilon) \in T_{d}$ for some $\mu \in Q_{d}$ whose domain is $B$ and where for every $q \in B,(q, u, \mu(q) s, \epsilon) \in T$.

This claim together with claim (0) and the definition of $\left.T_{d}\right|_{Q_{d, F}}$ show that $\mathcal{T}\left(M_{d}\right)=\mathcal{T}(M)$. Since $M_{d}$ is reduced deterministic by construction we are done provided Claims (0) through (4) hold.

Claims (0) through (4) must be proven together by induction on the depth of an input tree $u$. Note that termination of the construction is due to the second parts of claims (1) and (2).

Analogously to Theorem 9 we find:

Theorem 10. Assume $M$ is a reduced functional FST. Then the following three statements are equivalent:

(1) $\mathcal{T}(M)$ can be realized by a deterministic transducer.

(2) $M$ has Properties (wD0), (D1) and (D2).

(3) $M$ is of weakly bounded difference.

It can be decided whether or not the transduction of an FST can be realized by a deterministic one.

From Theorems 9 and Theorems 10 we conclude that the transduction of a deterministic transducer can be realized by a reduced deterministic one iff the corresponding reduced transducer has Property (D0). We have:

Corollary 11. Assume $M$ is a deterministic FST. Let $M_{r}$ be a reduced functional transducer with $\mathcal{T}(M)=\mathcal{T}\left(M_{r}\right)$. Then the following two statements are equivalent: 
(1) $\mathcal{T}(M)$ can be realized by a reduced deterministic transducer.

(2) $M_{r}$ has Property (DO).

It can be decided in polynomial time whether or not the transduction of a deterministic FST can be realized by a reduced deterministic one.

Proof. If $\mathcal{T}\left(M_{r}\right)$ can be realized by a reduced deterministic transducer then by Prop. $4, M_{r}$ has Property (D0). Conversely, assume $M_{r}$ has Property (D0). Since $\mathcal{T}\left(M_{r}\right)$ can be realized by a deterministic transducer (namely $M$ ), $M_{r}$ has Properties (D1) and (D2) by Prop. 6 and 8. But then by Theorem 9, $\mathcal{T}\left(M_{r}\right)$ can be realized by a reduced deterministic transducer.

However, the general construction of the equivalent reduced deterministic transducer given in the proof of Theorem 9 can be replaced by a simpler construction here. Let $M_{r}=\left(A_{r}, T_{r}\right)$ be the reduced functional transducer with $\mathcal{T}\left(M_{r}\right)=\mathcal{T}(M)$ as constructed in the proof of 2. Since $M$ was deterministic the set $D_{r}(u)$ of states derivable by some input tree $u$ (w.r.t. $A_{r}$ ) always is a subset of $\{\langle q, 0\rangle,\langle q, 1\rangle\}$ where $(q, u, \epsilon) \in \delta$. Therefore, if $\langle q, 0\rangle \notin D_{r}(u)$ we safely can produce output whereas as long as $\langle q, 0\rangle \in D_{r}(u)$ we only produce $\perp$ and memorize a possible output provided $\langle q, 1\rangle \in D_{r}(u)$.

To implement this idea assume $M=(A, T)$ with $A=\left(Q, \Sigma, \delta, Q_{F}\right)$. We construct $M_{d}=\left(A_{d}, T_{d}\right)$ with $A_{d}=\left(Q_{d}, \Sigma, \delta_{d}, Q_{d, F}\right)$ as follows. $Q_{d}$ is some set of pairs $Q \times\left(\{\perp\} \cup T_{\Delta} \cup\left\{x_{1}\right\}\right)$ such that

1. $\langle q, \perp\rangle \in Q_{d}$ iff $\langle q, 0\rangle \in Q_{r}$ but $\langle q, 1\rangle \notin Q_{r}$; i.e., the output for the presently processed subtree is irrelevant in all cases;

2. $\langle q, y\rangle \in Q_{d}$ with $y \in T_{\Delta}$ iff both $\langle q, 0\rangle \in Q_{r}$ and $\langle q, 1\rangle \in Q_{r}$; i.e., the output $t$ produced so far possibly is relevant; whereas

3. $\left\langle q, x_{1}\right\rangle \in Q_{d}$ iff $\langle q, 0\rangle \notin Q_{r}$ but $\langle q, 1\rangle \in Q_{r}$; i.e., the output is definitively relevant and already has been produced.

$Q_{d, F}$ consists of all pairs $\langle q, y\rangle \in Q_{d}$ where $q \in Q_{F}$, and $U\left(M_{d}\right)=Q_{d} \cap(Q \times$ $\left.\left(\{\perp\} \cup T_{\Delta}\right)\right)$.

The sets $Q_{d}$ and $\delta_{d}$ are again iteratively defined as the union of their "approximations", i.e., by

$$
Q_{d}=\bigcup_{\nu \geq 0} Q^{(\nu)} \text { and } \delta_{d}=\bigcup_{\nu \geq 0} \delta^{(\nu)}
$$

where $Q^{(0)}=\emptyset$ and $\delta^{(0)}=\emptyset$.

Assume $\nu>0$ and $\left\langle q_{1}, y_{1}\right\rangle, \ldots,\left\langle q_{k}, y_{k}\right\rangle \in Q^{(\nu-1)}$. Let $\tau=\left(q, a, q_{1} \ldots q_{k}\right) \in \delta$. We construct some $y \in\{\perp\} \cup T_{\Delta} \cup\left\{x_{1}\right\}$ such that $\langle q, y\rangle \in Q^{(\nu)}$ and $\tau_{d}=$ $\left(\langle q, y\rangle, a,\left\langle q_{1}, y_{1}\right\rangle \ldots\left\langle q_{k}, y_{k}\right\rangle\right) \in \delta^{(\nu)}$, and define $T_{d}\left(\tau_{d}\right)$.

Case 1: $\langle q, 0\rangle \in Q_{r}$ and $\langle q, 1\rangle \notin Q_{r}$.

Note that then also for every $j,\left\langle q_{j}, 0\right\rangle \in Q_{r}$ and $\left\langle q_{j}, 1\right\rangle \notin Q_{r}$. Hence, all $y_{j} \in\{\perp\} \cup T_{\Delta}$. Therefore, we put $y=\perp$ and $T_{d}\left(\tau_{d}\right)=\perp$.

Case $2:\langle q, 0\rangle \in Q_{r}$ and $\langle q, 1\rangle \in Q_{r}$.

Again, $y_{j} \in\{\perp\} \cup T_{\Delta}$ for all $j$. Moreover, $y_{j} \neq \perp$ whenever $x_{j}$ occurs in $T(\tau)$. Therefore, $y=T(\tau)\left[y_{1}, \ldots, y_{k}\right]$ in deed is in $T_{\Delta}$. Finally, we put $T_{d}\left(\tau_{d}\right)=\perp$. 
Case 3: $\langle q, 0\rangle \notin Q_{r}$ and $\langle q, 1\rangle \in Q_{r}$.

This is the only case where we have to produce an output pattern different from $\perp$. Define $y_{j}^{\prime}=x_{j}$ if $y_{j}=x_{1}$ and $y_{j}^{\prime}=y_{j}$ otherwise. Then put $y=x_{1}$ and $T_{d}\left(\tau_{d}\right)=T(\tau)\left[y_{1}^{\prime}, \ldots, y_{k}^{\prime}\right]$.

It is easy to verify that the construction terminates, and that this construction produces a reduced deterministic transducer which is equivalent to $M$ (or $\left.M_{r}\right)$. Especially for every $\langle q, y\rangle \in Q_{d}, \operatorname{depth}(y)<\# Q \cdot|M|$.

\section{Conclusion}

We gave a characterization of functional tree transducers whose transduction can be realized by deterministic or even reduced deterministic transducers. It remained open whether or not these two characterizations can be decided even in polynomial time. At least we were able to prove that it can be decided in polynomial time whether or not the transduction of a deterministic transducer can be realized by a reduced deterministic one.

\section{References}

1. A.V. Aho, J.E. Hopcroft, J.D. Ullman: The design and analysis of computer algorithms. Addison-Wesley, 1974

2. J. Berstel: Transductions and Context-Free Languages. Teubner, Stuttgart, 1979

3. J. Engelfriet: Some open questions and recent results on tree transducers and tree languages. In: Formal Language Theory, ed. by R.V. Book, Academic Press 1980, pp. $241-286$

4. Chr. Ferdinand, H. Seidl, R. Wilhelm: Tree automata for code selection. In: R. Giegerich, S.L. Graham (eds): Code Generation - Concepts, Tools, Techniques. Springer, Workshops in Computing, pp. 31-50, 1992

5. F. Gecseg, M. Steinby: Tree automata. Akademiai Kiado, Budapest, 1984

6. R. Giegerich, K. Schmal: Code selection techniques: pattern matching, tree parsing and inversion of derivors. Proc. of ESOP 1988, LNCS 300 pp. 245-268

7. C. Reutenauer: Subsequential Functions: Characterizations, Minimization, Examples. Proc. IMYCS 1990, LNCS 464, pp. 62-79

8. H. Seidl: Equivalence of finite-valued bottom-up finite state tree transducers is decidable. Proc. CAAP '90, LNCS 431, pp. 269-284, 1990; extended version to appear in: Math. Syst. Theory

9. H. Seidl: Single-valuedness of tree transducers is decidable in polynomial time. To appear in: TCS, special issue of CAAP 90

10. A. Weber, R. Klermm:: Economy of Description for Single-valued Transducers. Preprint J.W.Goethe-Universität, Frankfurt/Main, 1991

11. Z. Zachar: The solvability of the equivalence problem for deterministic frontierto-root tree transducers. Acta Cybernetica 4 (1978), pp. 167-177 